\title{
Tempered Generalized Functions and Hermite Expansions
}

\author{
Pedro Catuognd 1 and Christian Olivera 2 \\ Departamento de Matemática, Universidade Estadual de Campinas, \\ F. 54(19) 3521-5921 Fax 54(19) 3521-6094 \\ 13.081-970 - Campinas - SP, Brazil.e-mail: pedrojc@ime.unicamp.br ; \\ colivera@ime.unicamp.br
}

\begin{abstract}
In this work we introduce a new algebra of tempered generalized functions. The tempered distributions are embedded in this algebra via their Hermite expansions. The Fourier transform is naturally extended to this algebra in such a way that the usual relations involving multiplication, convolution and differentiation are valid. We study the elementary properties of the association, embedding, point values and Fourier transform. Furthermore, we give a generalized Itô formula in this context and some applications to stochastic analysis.
\end{abstract}

Key words: Hermite functions, Generalized function algebras, Fourier transform, Itô formula.

MSC2000 subject classification: 46F30, $60 \mathrm{H}$.

\footnotetext{
${ }^{1}$ Research partially supported by CNPQ 302704/2008-6.

${ }^{2}$ Research partially supported by FAPESP 02/10246-2, 2007/54740-4.
} 


\section{Introduction}

The differential algebras of generalized functions of Colombeau type were developed in connection with non linear problems. These algebras provide a good framework to solve differential equations with rough initial data or discontinuous coefficients (see [3], [9] and [13]). Recently there has been a great interest in developing a stochastic calculus in algebras of generalized functions (see [1], 44, [11], [12], [14] and [20] and references).

In order to develop a Fourier transform for generalized functions, J. F. Colombeau in [5] introduced an algebra of tempered generalized functions with the characteristic that most of the properties involving the Fourier transform and convolution are valid only in a weak sense. For a recent account of the theory and applications we refer to the reader to [7] and [9].

The following question naturally arises: is it possible to construct an algebra of generalized functions in which the operations of multiplication, convolution, differentiation and Fourier transform are defined and have all the properties of the ordinary operations?

In this work we introduced a new algebra of tempered generalized functions that answers the above question. The construction of this algebra is based on the Fourier-Hermite expansion of tempered distributions. More precisely, the Hermite representation theorem for $\mathcal{S}^{\prime}$ (see [2], [18, [22], and [23]) establishes that every $S \in \mathcal{S}^{\prime}$ can be represented by a Hermite series

$$
S=\sum_{j=0}^{\infty} S\left(h_{j}\right) h_{j}
$$

where $\left\{h_{j}\right\}$ are the Hermite functions and the equality is in the weak sense. The idea is to embed the tempered distributions into a differential subalgebra $\mathcal{H}_{\mathbf{s}^{\prime}} \subset \mathcal{S}^{\mathbb{N}_{0}}$ via the sequence of partial sums

$$
S_{n}=\sum_{j=0}^{n} S\left(h_{j}\right) h_{j}
$$


and define the algebra of tempered generalized functions as

$$
\mathcal{H}=\mathcal{H}_{\mathrm{s}^{\prime}} / \mathcal{H}_{\mathrm{s}}
$$

where $\mathcal{H}_{\mathrm{s}}$ is a differentiable ideal of $\mathcal{H}_{\mathbf{s}^{\prime}}$ (see Section 3 for precise definitions and details).

The operations in $\mathcal{H}$ are naturally introduced in a pointwise way. By example the Fourier transform of $\left[f_{n}\right] \in \mathcal{H}$ is defined to be $\left[\mathcal{F}\left(f_{n}\right)\right]$, where $\mathcal{F}$ is the usual Fourier transform. Now, it is not difficult to prove that the Fourier transform is an isomorphism and that usual identities involving convolution, derivatives and Fourier transform are satisfied.

Another important point of this paper is that we obtain an Itô formula under weak regularity conditions. We make use of Föllmer's formulation of pathwise stochastic calculus [8] in order to establish an Itô formula for tempered generalized functions. This formula extends the classical one via association and the embedding of the tempered distributions into $\mathcal{H}$.

The paper is organized as follows: Section 2 contains a brief summary without proofs of Hermite functions and the Hermite representation theorems. In section 3 , we introduce the tempered algebra $\mathcal{H}$, this algebra contains the tempered distributions and extends the product in $\mathcal{S}$. Moreover, we study some elementary properties and we show that the symmetric Hermite product of tempered distributions (see [6] and [24]) is associated with the product in $\mathcal{H}$.

In section 4 , in order to define the point value of tempered generalized functions, we introduce the ring of tempered numbers $\mathbf{h}$. Section 5 we deal with integration, convolutions and the Fourier transform of tempered generalized functions. We obtain the Fourier inversion theorem for $\mathcal{H}$, the formula of interchange between the product and the convolution and the rule of integration by parts. We remark that the identities are in $\mathbf{h}$, this is in a strong sense. 
In section 6 we introduce an elementary stochastic calculus for generalized tempered functions. We derive an Itô formula for elements of $\mathcal{H}$. We observe that the classical Itô formula is associated to the generalized Itô formula, under suitable regularity conditions. Moreover, we obtain a probabilistic representation of the solution of the heat equation with data in $\mathcal{H}$. Finally we note that in [10] and [11] is present an Itô formula for generalized functions containing some mistakes in definitions and proofs; see [4].

\section{N-Representation of tempered distributions.}

Let $\mathcal{S} \equiv \mathcal{S}(\mathbb{R})$ be the Schwartz space of rapidly decreasing smooth real valued functions.

For each $m \in \mathbb{N}_{0} \equiv \mathbb{N} \cup\{0\}$, we consider $\|\cdot\|_{m}$ the norm of $\mathcal{S}$ given by

$$
\|\varphi\|_{m}=\left(\int_{-\infty}^{\infty}\left|(N+1)^{m} \varphi(x)\right|^{2} d x\right)^{\frac{1}{2}}
$$

where $N+1=\frac{1}{2}\left(-\frac{d^{2}}{d x^{2}}+x^{2}+1\right)$.

We observe that $\mathcal{S}$ is provided with the natural topology given by these norms is a sequentially complete locally convex space and its dual space $\mathcal{S}^{\prime}$ is the space of tempered distributions. The family of norms $\left\{\|\cdot\|_{m}: m \in \mathbb{N}_{0}\right\}$ is direct and equivalent to the family of seminorms $\left\{\|\cdot\|_{\alpha, \beta, \infty}: \alpha, \beta \in \mathbb{N}_{0}\right\}$, given by

$$
\|\varphi\|_{\alpha, \beta, \infty}=\sup _{x}\left|\left(1+|x|^{2}\right)^{\alpha} D^{\beta} \varphi(x)\right| .
$$

We use often the following property of the multiplication on $\mathcal{S}$. For all $m \in \mathbb{N}_{0}$ there exists $r, s \in \mathbb{N}_{0}$ and a constant $C_{m}>0$ such that

$$
\|\varphi \psi\|_{m} \leq C_{m}\|\varphi\|_{r}\|\psi\|_{s}
$$

for all $\varphi, \psi \in \mathcal{S}$ (see for instance [16], Theorem 2).

The Hermite polynomials $H_{n}(x)$ are defined by 


$$
H_{n}(x)=(-1)^{n} e^{\frac{x^{2}}{2}} \frac{d^{n}}{d x^{n}} e^{-\frac{x^{2}}{2}}
$$

for $n \in \mathbb{N}_{0}$ or equivalently

$$
H_{n}(x)=2^{-\frac{n}{2}} \sum_{k=0}^{[n / 2]} \frac{(-1)^{k} n !(\sqrt{2} x)^{n-2 k}}{k !(n-2 k) !} .
$$

The Hermite functions $h_{n}(x)$ are defined by

$$
h_{n}(x)=(\sqrt{2 \pi} n !)^{-\frac{1}{2}} e^{-\frac{1}{4} x^{2}} H_{n}(x)
$$

for $n \in \mathbb{N}_{0}$. Some properties of the Hermite functions that we will often use are:

- $h_{n} \in \mathcal{S}$ for all $n \in \mathbb{N}_{0}$,

- $h_{n}$ is an even (odd) function if $n$ is even (odd),

- $\sqrt{n+1} h_{n+1}(x)+2 h_{n}^{\prime}(x)=\sqrt{n} h_{n-1}(x)$ for all $n \in \mathbb{N}_{0}$,

- $\sqrt{n+1} h_{n+1}(x)=x h_{n}(x)-\sqrt{n} h_{n-1}(x)$ for all $n \in \mathbb{N}_{0}$,

- $\left\{h_{n}: n \in \mathbb{N}_{0}\right\}$ is an orthonormal basis of $L^{2}(\mathbb{R})$,

- $(N+1) h_{n}=(n+1) h_{n}$ for all $n \in \mathbb{N}_{0}$.

From the two last properties we have

$$
\|\varphi\|_{m}^{2}=\sum_{n=0}^{\infty}(n+1)^{2 m}<\varphi, h_{n}>^{2},
$$

where $\left\langle\varphi, h_{n}\right\rangle=\int \varphi(x) h_{n}(x) d x$ are the Fourier-Hermite coefficients of the expansion of $\varphi$.

The Hermite representation theorem for $\mathcal{S}\left(\mathcal{S}^{\prime}\right)$ states a topological isomorphism from $\mathcal{S}\left(\mathcal{S}^{\prime}\right)$ onto the space of sequences $\mathbf{s}\left(\mathbf{s}^{\prime}\right)$. 
Let $\mathbf{s}$ be the space of rapidly decreasing sequences

$$
\mathbf{s}=\left\{\left(a_{n}\right) \in \ell^{2}: \sum_{n=0}^{\infty}(n+1)^{2 m}\left|a_{n}\right|^{2}<\infty, \text { for all } m \in \mathbb{N}_{0}\right\} .
$$

The space $\mathbf{s}$ is a locally convex space with the sequence of norms

$$
\left\|\left(a_{n}\right)\right\|_{m}=\left(\sum_{n=0}^{\infty}(n+1)^{2 m}\left|a_{n}\right|^{2}\right)^{\frac{1}{2}}
$$

or with the equivalent sequence of norms

$$
\left|\left(a_{n}\right)\right|_{m, \infty}=\sup _{n}(n+1)^{m}\left|a_{n}\right| .
$$

The topological dual space to $\mathbf{s}$, denoted by $\mathbf{s}^{\prime}$, is given by

$$
\mathbf{s}^{\prime}=\left\{\left(b_{n}\right): \text { for some }(C, m) \in \mathbb{R} \times \mathbb{N}_{0},\left|b_{n}\right| \leq C(n+1)^{m} \text { for all } n\right\} .
$$

The natural pairing of elements from $\mathbf{s}$ and $\mathbf{s}^{\prime}$, denoted by $\langle\cdot, \cdot\rangle$, is given by

$$
\left\langle\left(b_{n}\right),\left(a_{n}\right)\right\rangle=\sum_{n=0}^{\infty} b_{n} a_{n}
$$

for $\left(b_{n}\right) \in \mathbf{s}^{\prime}$ and $\left(a_{n}\right) \in \mathbf{s}$.

It is clear that $\mathbf{s}^{\prime}$ is an algebra with the pointwise operations:

$$
\begin{aligned}
\left(b_{n}\right)+\left(b_{n}^{\prime}\right) & =\left(b_{n}+b_{n}^{\prime}\right) \\
\left(b_{n}\right) \cdot\left(b_{n}^{\prime}\right) & =\left(b_{n} b_{n}^{\prime}\right),
\end{aligned}
$$

and $\mathbf{s}$ is an ideal of $\mathbf{s}^{\prime}$.

The relation between $\mathbf{s}\left(\mathbf{s}^{\prime}\right)$ and $\mathcal{S}\left(\mathcal{S}^{\prime}\right)$ is induced by the Hermite functions, via Hermite coefficients (evaluation). The following representation theorem is fundamental in our work, for the proof see [18] pp. 143.

Theorem 2.1 (N-representation theorem for $\mathcal{S}$ and $\mathcal{S}^{\prime}$ ) a) Let $\mathbf{h}: \mathcal{S} \rightarrow$ $\mathbf{s}$ be the application

$$
\mathbf{h}(\varphi)=\left(<\varphi, h_{n}>\right)
$$


Then $\mathbf{h}$ is a topological isomorphism. Moreover,

$$
\|\mathbf{h}(\varphi)\|_{m}=\|\varphi\|_{m}
$$

for all $\varphi \in \mathcal{S}$.

b) Let $\mathbf{H}: \mathcal{S}^{\prime} \rightarrow \mathbf{s}^{\prime}$ be the application $\mathbf{H}(T)=\left(T\left(h_{n}\right)\right)$. Then $\mathbf{H}$ is a topological isomorphism. Moreover, if $T \in \mathcal{S}^{\prime}$ we have that

$$
T=\sum_{n=0}^{\infty} T\left(h_{n}\right) h_{n}
$$

in the weak sense and for all $\varphi \in \mathcal{S}$,

$$
T(\varphi)=\langle\mathbf{H}(T), \mathbf{h}(\varphi)\rangle .
$$

We say that the sequences $\mathbf{h}(\varphi)$ and $\mathbf{H}(T)$ are the Hermite coefficients of the tempered function $\varphi$ and the distribution $T$, respectively.

Next we provide the Hermite coefficients for some tempered distributions.

\subsection{The delta distribution.}

(see 2] pp 191.)

$$
\delta\left(h_{n}\right)=h_{n}(0)= \begin{cases}\frac{(-1)^{\frac{n}{2}}}{\sqrt[4]{2 \pi}} \sqrt{\frac{13}{2} \frac{3}{4} \cdots \frac{n-1}{n}} & \text { for } n \text { even } \\ 0 & \text { for } n \text { odd }\end{cases}
$$

\subsection{The constant distribution 1 .}

(see [2] pp 190.)

$$
1\left(h_{n}\right)=\int_{-\infty}^{\infty} h_{n}(x) d x= \begin{cases}\sqrt[4]{8 \pi} \sqrt{\frac{13}{2} \frac{3}{4} \cdots \frac{n-1}{n}} & \text { for } n \text { even } \\ 0 & \text { for } n \text { odd }\end{cases}
$$




\subsection{The $x_{+}^{p}$ distribution.}

(see [21] pp 162.)

We recall that $\left\langle x_{+}^{p}, \phi>=\int_{0}^{\infty} x^{p} \phi(x) d x\right.$.

$$
x_{+}^{p}\left(h_{n}\right)= \begin{cases}(\sqrt{2 \pi} n !)^{-\frac{1}{2}} 2^{p} \Gamma\left(\frac{p+1}{2}\right) W_{n}(2 p+1) & \text { for } n \text { even } \\ (\sqrt{2 \pi} n !)^{-\frac{1}{2}} 2^{p+1} \Gamma\left(\frac{p+2}{2}\right) W_{n}(2 p+1) & \text { for } n \text { odd }\end{cases}
$$

where $W_{n}(x)$ are polynomials such that $W_{0}(x)=W_{1}(x)=1$ and

$$
W_{n+2}(x)=x W_{n}(x)+n(n-1) W_{n-2}(x) .
$$

Note that if $p=0$, then $x_{+}^{p}$ is the Heaviside distribution $H$.

\subsection{The $\delta^{\prime}$ distribution.}

$$
\delta^{\prime}\left(h_{n}\right)=-h_{n}^{\prime}(0)=\sqrt{n} h_{n-1}(0) .
$$

\section{The Tempered Algebra}

In order to introduce the tempered algebra we consider $\mathcal{S}^{\mathbb{N}_{0}}$ the space of sequences of rapidly decreasing smooth functions. It is clear that $\mathcal{S}^{\mathbb{N}_{0}}$ has the structure of an associative, commutative differential algebra with the natural operations:

$$
\begin{aligned}
\left(f_{n}\right)+\left(g_{n}\right) & =\left(f_{n}+g_{n}\right) \\
a\left(f_{n}\right) & =\left(a f_{n}\right) \\
\left(f_{n}\right) \cdot\left(g_{n}\right) & =\left(f_{n} g_{n}\right) \\
D\left(f_{n}\right) & =\left(D f_{n}\right)
\end{aligned}
$$

where $\left(f_{n}\right)$ and $\left(g_{n}\right)$ are in $\mathcal{S}$ and $a \in \mathbb{R}$. 
Definition 3.1 Let

$$
\mathcal{H}_{\mathbf{s}^{\prime}}=\left\{\left(f_{n}\right) \in \mathcal{S}^{\mathbb{N}_{0}}: \text { for each } m \in \mathbb{N}_{0},\left(\left\|f_{n}\right\|_{m}\right) \in \mathbf{s}^{\prime}\right\}
$$

and

$$
\mathcal{H}_{\mathbf{s}}=\left\{\left(f_{n}\right) \in \mathcal{S}^{\mathbb{N}_{0}}: \text { for each } m \in \mathbb{N}_{0},\left(\left\|f_{n}\right\|_{m}\right) \in \mathbf{s}\right\}
$$

Lemma $3.1 \mathcal{H}_{\mathbf{s}^{\prime}}$ is a subalgebra of $\mathcal{S}^{\mathbb{N}_{0}}$ and $\mathcal{H}_{\mathbf{s}}$ is a differential ideal of $\mathcal{H}_{\mathbf{s}^{\prime}}$.

Proof: Let $\left(f_{n}\right),\left(g_{n}\right) \in \mathcal{H}_{\mathbf{s}^{\prime}}$ and $m \in \mathbb{N}_{0}$. Applying the inequality (2), there exists $r, s \in \mathbb{N}_{0}$ and a constant $C_{m}>0$ such that

$$
\left\|f_{n} g_{n}\right\|_{m} \leq C_{m}\left\|f_{n}\right\|_{r}\left\|g_{n}\right\|_{s}
$$

By definition, there exists constants $D, E>0$ and $p, q \in \mathbb{N}_{0}$ such that

$$
\begin{aligned}
\left\|f_{n}\right\|_{r} & \leq D(n+1)^{p} \\
\left\|g_{n}\right\|_{s} & \leq E(n+1)^{q} .
\end{aligned}
$$

Combining these inequalities, we obtain

$$
\left\|f_{n} g_{n}\right\|_{m} \leq C_{m} D E(n+1)^{p+q}
$$

This proves that $\left(\left\|f_{n} g_{n}\right\|_{m}\right) \in \mathbf{s}^{\prime}$, thus $\left(f_{n}\right) \cdot\left(g_{n}\right) \in \mathcal{H}_{\mathbf{s}^{\prime}}$.

Now, we prove that $\mathcal{H}_{\mathbf{s}}$ is an ideal of $\mathcal{H}_{\mathbf{s}^{\prime}}$. Let $\left(f_{n}\right) \in \mathcal{H}_{\mathbf{s}^{\prime}},\left(g_{n}\right) \in \mathcal{H}_{\mathbf{s}}$ and $m \in \mathbb{N}_{0}$. From (3.1) we have that for each $r \in \mathbb{N}_{0}$ there exists a constant $D>0$ and $p \in \mathbb{N}_{0}$ such that

$$
\left\|f_{n}\right\|_{r} \leq D(n+1)^{p}
$$

and for all $s, l \in \mathbb{N}_{0}$,

$$
\left\|\left(\left\|g_{n}\right\|_{s}\right)\right\|_{l}^{2}=\sum_{n=0}^{\infty}(n+1)^{2 l}\left\|g_{n}\right\|_{s}^{2}<\infty .
$$


Combining the inequality (2) with the above equations we obtain

$$
\begin{aligned}
\left\|\left(\left\|f_{n} g_{n}\right\|_{m}\right)\right\|_{l}^{2} & =\sum_{n=0}^{\infty}(n+1)^{2 l}\left\|f_{n} g_{n}\right\|_{m}^{2} \\
& \leq C_{m}^{2} \sum_{n=0}^{\infty}(n+1)^{2 l}\left\|f_{n}\right\|_{r}^{2}\left\|g_{n}\right\|_{s}^{2} \\
& \leq C_{m}^{2} D^{2} \sum_{n=0}^{\infty}(n+1)^{2(l+p)}\left\|g_{n}\right\|_{s}^{2} \\
& <\infty .
\end{aligned}
$$

Note that we have proved that $\left(\left\|f_{n} g_{n}\right\|_{m}\right) \in \mathbf{s}$, for all $m \in \mathbb{N}_{0}$, therefore $\left(f_{n}\right) \cdot\left(g_{n}\right) \in \mathcal{H}_{\mathbf{s}}$.

Finally, we prove that if $\left(f_{n}\right) \in \mathcal{H}_{\mathbf{s}}$ then $\left(D f_{n}\right) \in \mathcal{H}_{\mathbf{s}}$. In fact, let $m \in \mathbb{N}_{0}$. Since $\left\{\|\cdot\|_{m}: m \in \mathbb{N}_{0}\right\}$ is equivalent to $\left\{\|\cdot\|_{\alpha, \beta, \infty}: \alpha, \beta \in \mathbb{N}_{0}\right\}$ we have that there exists $\alpha, \beta, m_{\alpha, \beta} \in \mathbb{N}_{0}$ and a constants $C_{m}, C_{\alpha, \beta+1}>0$ such that

$$
\begin{aligned}
\left\|D f_{n}\right\|_{m} & \leq C_{m}\left\|D f_{n}\right\|_{\alpha, \beta, \infty} \\
& =C_{m}\left\|f_{n}\right\|_{\alpha, \beta+1, \infty} \\
& \leq C_{m} C_{\alpha, \beta+1}\left\|f_{n}\right\|_{m_{\alpha, \beta}} .
\end{aligned}
$$

As $\left(f_{n}\right) \in \mathcal{H}_{\mathbf{s}}$ we have $\left(\left\|D f_{n}\right\|_{m}\right) \in \mathbf{s}$, this implies that $\left(D f_{n}\right) \in \mathcal{H}_{\mathbf{s}}$.

Proposition 3.1 Let $T \in \mathcal{S}^{\prime}$. Then $\left(T_{n}\right) \in \mathcal{H}_{\mathbf{s}^{\prime}}$, where $T_{n}=\sum_{j=0}^{n} T\left(h_{j}\right) h_{j}$.

Proof: From Theorem 2.1, there exists a constant $C>0$ and $p \in \mathbb{N}_{0}$ such that

$$
\left|T\left(h_{j}\right)\right| \leq C(j+1)^{p}
$$

for all $j \in \mathbb{N}_{0}$. Then

$$
\left\|T_{n}\right\|_{m}^{2}=\sum_{j=0}^{n}(j+1)^{2 m}\left|T\left(h_{j}\right)\right|^{2}
$$




$$
\begin{aligned}
& \leq(n+1)^{2 m} \sum_{j=0}^{n}\left|T\left(h_{j}\right)\right|^{2} \\
& \leq C(n+1)^{2(m+p+1)} .
\end{aligned}
$$

This completes the proof.

Definition 3.2 The tempered algebra is defined as

$$
\mathcal{H}=\mathcal{H}_{\mathbf{s}^{\prime}} / \mathcal{H}_{\mathrm{s}}
$$

The elements of $\mathcal{H}$ are called tempered generalized functions.

Let $\left(f_{n}\right) \in \mathcal{H}_{s^{\prime}}$ we will use $\left[f_{n}\right]$ to denote the equivalent class $\left(f_{n}\right)+\mathcal{H}_{s}$.

Proposition 3.2 Let $\iota: \mathcal{S}^{\prime} \rightarrow \mathcal{H}$ be the application

$$
\iota(T)=\left[T_{n}\right]
$$

Then $\iota$ is a linear embedding. Moreover, we have that

a) For all $\varphi \in \mathcal{S}$,

$$
\iota(\varphi)=[\varphi]
$$

b) For all $T \in \mathcal{S}^{\prime}$

$$
\iota(D T)=D \iota(T)
$$

Proof: It is clear from the previous Proposition, that $\iota$ is well defined and a linear application. We claim that $\iota(T)=0$ implies $T=0$. Since $\left(T_{n}\right) \in \mathcal{H}_{\mathbf{s}}$, we have

$$
\lim _{n \rightarrow \infty}\left\|T_{n}\right\|_{m}=0
$$

for all $m \in \mathbb{N}_{0}$, therefore the sequence $\left(T_{n}\right)$ converges weakly to 0 , which proves the claim. 
a) Let $\varphi \in \mathcal{S}$. We have that $\iota(\varphi)=\left[\varphi_{n}\right]$ where $\varphi_{n}=\sum_{j=0}^{n}<\varphi, h_{j}>h_{j}$. Then for all $m, s \in \mathbb{N}_{0}$ we have that

$$
\begin{aligned}
\lim _{n \rightarrow \infty}(n+1)^{2 s}\left\|\varphi-\varphi_{n}\right\|_{m}^{2} & =\lim _{n \rightarrow \infty}(n+1)^{2 s} \sum_{j=n+1}^{\infty}(j+1)^{2 m}\left|<\varphi, h_{j}>\right|^{2} \\
& \leq \lim _{n \rightarrow \infty} \sum_{j=n+1}^{\infty}(j+1)^{2(m+s)}\left|<\varphi, h_{j}>\right|^{2} \\
& =0
\end{aligned}
$$

where the last equality follows from $\|\varphi\|_{m+s}<\infty$. Therefore, we conclude that $\left(\left\|\varphi-\varphi_{n}\right\|_{m}\right) \in \mathbf{s}$. Since $\left(\varphi-\varphi_{n}\right) \in \mathcal{H}_{\mathbf{s}}$, it follows that $\iota(\varphi)=[\varphi]$.

b) Let $T \in \mathcal{S}^{\prime}$. Using the above definitions and properties of Hermite functions it follows that:

$$
\begin{aligned}
D T_{n} & =D\left(\sum_{j=0}^{n} T\left(h_{j}\right) h_{j}\right) \\
& =\sum_{j=0}^{n} T\left(h_{j}\right) D h_{j} \\
& =\sum_{j=0}^{n} T\left(h_{j}\right) \frac{1}{2}\left(\sqrt{j} h_{j-1}-\sqrt{j+1} h_{j+1}\right) \\
& =-\sum_{j=0}^{n} \frac{1}{2}\left(\sqrt{j} T\left(h_{j-1}\right)-\sqrt{j+1} T\left(h_{j+1}\right)\right) h_{j} \\
& =\sum_{j=0}^{n} D T\left(h_{j}\right) h_{j} \\
& =(D T)_{n} .
\end{aligned}
$$

Therefore $D \iota(T)=\left[D T_{n}\right]=\left[(D T)_{n}\right]=\iota(D T)$.

Corollary 3.3 Let $\varphi, \psi \in \mathcal{S}$. Then

$$
\iota(\varphi \psi)=\iota(\varphi) \cdot \iota(\psi)
$$


Proof: We first observe that

$$
(\varphi \psi)-\left(\varphi_{n}\right) \cdot\left(\psi_{n}\right)=(\varphi) \cdot\left(\psi-\psi_{n}\right)+\left(\varphi-\varphi_{n}\right) \cdot(\psi)
$$

Applying Proposition 3.2 and Lemma 3.1 we obtain $(\varphi \psi)-\left(\varphi_{n}\right) \cdot\left(\psi_{n}\right) \in \mathcal{H}_{\mathbf{s}}$. Therefore $\iota(\varphi \psi)=\iota(\varphi) \cdot \iota(\psi)$.

Remark 3.1 Let $\mathcal{O}_{M}$ be the ring of multipliers of $\mathcal{S}$ (see [18]). We have a natural multiplication from $\mathcal{O}_{M}$ by $\mathcal{H}$ into $\mathcal{H}$, defined by

$$
g\left[f_{n}\right]:=\left[g f_{n}\right]
$$

where $g \in \mathcal{O}_{M}$ and $\left[f_{n}\right] \in \mathcal{H}$.

It is easy to check that the product is well defined and that $\mathcal{H}$ is a $\mathcal{O}_{M}$-module.

Now, we give two examples of tempered generalized functions.

Example 3.1 The distribution $\delta$. We have that $\iota(\delta)=\left[\delta_{n}\right]$, where $\delta_{n}=$ $\sum_{j=0}^{n} h_{j}(0) h_{j}$. Applying the formula (6) and the following equality

$$
\sum_{j=0}^{n} h_{j}(x) h_{j}(y)=\frac{\sqrt{n+1}}{x-y}\left(h_{n+1}(x) h_{n}(y)-h_{n+1}(y) h_{n}(x)\right),
$$

we see that

$$
\delta_{n}(x)= \begin{cases}\sqrt{n+1} \frac{(-1)^{\frac{n}{2}}}{\sqrt[4]{2 \pi}} \sqrt{\frac{1}{2} \frac{3}{4} \cdots \frac{n-1}{n}} \frac{h_{n+1}(x)}{x} & \text { for } n \text { even } \\ \sqrt{n+1} \frac{(-1)^{\frac{n+3}{2}}}{\sqrt[4]{2 \pi}} \sqrt{\frac{1}{2} \frac{3}{4} \cdots \frac{n}{n+1}} \frac{h_{n}(x)}{x} & \text { for } n \text { odd } .\end{cases}
$$

Example 3.2 The element $\delta^{2}$. We have that $\delta^{2} \equiv \iota(\delta) \cdot \iota(\delta)=\left[\delta_{n}^{2}\right]$. From (12) it follows that

$$
\delta_{n}^{2}(x)=\left\{\begin{array}{lll}
(n+1) \frac{1}{\sqrt{2 \pi}}\left(\frac{1}{2} \frac{3}{4} \cdots \frac{n-1}{n}\right) & \frac{h_{n+1}^{2}(x)}{x^{2}} & \text { for } n \text { even }, \\
(n+1) \frac{1}{\sqrt{2 \pi}}\left(\frac{1}{2} \frac{3}{4} \cdots \frac{n}{n+1}\right) & \frac{h_{n}^{2}(x)}{x^{2}} & \text { for } n \text { odd } .
\end{array}\right.
$$


We introduce the concept of association for tempered generalized functions.

Definition 3.3 Let $\left[f_{n}\right]$ and $\left[g_{n}\right]$ be tempered generalized functions. We say that $\left[f_{n}\right]$ and $\left[g_{n}\right]$ are associated, denoted by $\left[f_{n}\right] \approx\left[g_{n}\right]$, if for all $\varphi \in \mathcal{S}$

$$
\lim _{n \rightarrow \infty}<f_{n}-g_{n}, \varphi>=0 .
$$

We observe that the relation $\approx$ is well defined. In fact, if $\left(l_{n}\right) \in \mathcal{H}_{\mathrm{s}}$ and $\varphi \in \mathcal{S}$ we have that $\lim _{n \rightarrow \infty}\left\langle l_{n}, \varphi\right\rangle=0$. It follows immediately that $\approx$ is an equivalence relation on $\mathcal{H}$.

Proposition 3.4 a) Let $\left[f_{n}\right],\left[g_{n}\right] \in \mathcal{H}$ such that $\left[f_{n}\right] \approx\left[g_{n}\right]$. Then $D^{\alpha}\left[f_{n}\right] \approx$ $D^{\alpha}\left[g_{n}\right]$ for all $\alpha \in \mathbb{N}$.

b) Let $\left[f_{n}\right],\left[g_{n}\right] \in \mathcal{H}$ such that $\left[f_{n}\right] \approx\left[g_{n}\right]$ and $l \in \mathcal{O}_{M}$. Then $l\left[f_{n}\right] \approx l\left[g_{n}\right]$.

c) Let $T, S \in \mathcal{S}^{\prime}$ such that $\iota(T) \approx \iota(S)$. Then $T=S$.

Proof: a) By integration by parts and hypothesis,

$$
\begin{aligned}
\lim _{n \rightarrow \infty}\left\langle D^{\alpha} f_{n}-D^{\alpha} g_{n}, \varphi>\right. & =\lim _{n \rightarrow \infty}<f_{n}-g_{n},(-1)^{\alpha} D^{\alpha} \varphi> \\
& =0
\end{aligned}
$$

for all $\varphi \in \mathcal{S}$, that is, $D^{\alpha}\left[f_{n}\right] \approx D^{\alpha}\left[g_{n}\right]$.

b) Let $\varphi \in \mathcal{S}$. As $l \in \mathcal{O}_{M}$ we have $l \varphi \in \mathcal{S}$. By assumption,

$$
\begin{aligned}
\lim _{n \rightarrow \infty}\left\langle l f_{n}-l g_{n}, \varphi\right\rangle & =\lim _{n \rightarrow \infty}<f_{n}-g_{n}, l \varphi> \\
& =0 .
\end{aligned}
$$

We conclude that $l\left[f_{n}\right] \approx l\left[g_{n}\right]$.

c) By definition, $\lim _{n \rightarrow \infty} \int(T-S)_{n}(x) h_{k}(x) d x=(T-S)\left(h_{k}\right)$ for all $k \in \mathbf{N}_{0}$. But $\lim _{n \rightarrow \infty} \int(T-S)_{n}(x) h_{k}(x) d x=0$ since $\iota(T) \approx \iota(S)$. Applying the Nrepresentation theorem we conclude that $T=S$.

Example $3.3 x \iota(\delta) \approx 0$. In fact,

$$
\lim _{n \rightarrow \infty}<x \delta_{n}, h_{k}>=\left(x h_{k}\right)(0)=0 .
$$


Finally, we are interested in the relation between the symmetric Hermite product of tempered distributions via Hermite expansions and the product of tempered generalized functions. The symmetric Hermite product of tempered distribution was introduced by C. Shen and M. Sun in [24] based on our previous work [6].

Definition 3.4 Let $S$ and $T$ be tempered distributions. Suppose that for all $k \in \mathbb{N} \cup\{0\}$ there exists

$$
c_{k}=\lim _{n \rightarrow \infty}<T_{n} S_{n}, h_{k}>
$$

and that $\left(c_{k}\right) \in \mathbf{s}^{\prime}$. The symmetric Hermite product of $S$ and $T$, denoted by $S \bullet T$, is defined to be the tempered distribution

$$
\sum_{k=0}^{\infty} c_{k} h_{k}
$$

Lemma 3.2 a) The symmetric Hermite product is commutative and distributive.

b) The symmetric Hermite product verifies the Leibnitz rule: Let $S$ and $T$ be in $\mathcal{S}^{\prime}$, then

$$
D(S \bullet T)=D S \bullet T+S \bullet D T .
$$

c) Let $S$ and $T$ be in $\mathcal{S}^{\prime}$ such that there exists $S \bullet T$. Then

$$
\iota(S) \cdot \iota(T) \approx \iota(S \bullet T) .
$$

Proof: a), b) The proofs are straightforward (see [6]).

c) It is immediate from the definitions.

Remark 3.2 In order to work with ordinary differential equations in the tempered generalized functions setting, we introduce the algebra $\mathcal{H}_{T}$ of time dependent tempered generalized functions. We can proceed in a similar way 
to the construction of the algebra $\mathcal{H}$, the details are left to the reader. Let $\mathcal{S}_{T}$ be the set of functions $f:[0, T] \times \mathbb{R} \rightarrow \mathbb{R}$ such that for each $t \in[0, T]$, $f(t, \cdot) \in \mathcal{S}$ and for each $x \in \mathbb{R}, f(\cdot, x) \in C^{1}([0, T])$. The set $\mathcal{H}_{\mathrm{s}^{\prime}}^{T}$ is given by $\left\{\left(f_{n}\right) \in \mathcal{S}_{T}^{\mathbb{N}_{0}}:\right.$ for each $\left.m \in \mathbb{N}_{0},\left(\sup _{t \in[0, T]}\left\|f_{n}(t, \cdot)\right\|_{m}\right),\left(\sup _{t \in[0, T]}\left\|\frac{\partial f_{n}}{\partial t}(t, \cdot)\right\|_{m}\right) \in \mathbf{s}^{\prime}\right\}$ and the set $\mathcal{H}_{\mathrm{s}}^{T}$ is given by $\left\{\left(f_{n}\right) \in \mathcal{S}_{T}^{\mathbb{N}_{0}}:\right.$ for each $\left.m \in \mathbb{N}_{0},\left(\sup _{t \in[0, T]}\left\|f_{n}(t, \cdot)\right\|_{m}\right),\left(\sup _{t \in[0, T]}\left\|\frac{\partial f_{n}}{\partial t}(t, \cdot)\right\|_{m}\right) \in \mathbf{s}\right\}$.

It is clear that $\mathcal{H}_{\mathrm{s}}^{T}$ is a differentiable ideal of the algebra $\mathcal{H}_{\mathrm{s}^{\prime}}^{T}$. We define the algebra $\mathcal{H}_{T}$ as $\mathcal{H}_{\mathrm{s}^{\prime}}^{T} / \mathcal{H}_{\mathrm{s}}^{T}$. The elements of $\mathcal{H}_{T}$ are called time depended tempered generalized functions. It follows immediately that for $\left[f_{n}\right] \in \mathcal{H}_{T}$ we have that $\frac{\partial}{\partial t}\left[f_{n}(t, \cdot)\right]$ is define by $\left[\frac{\partial}{\partial t} f_{n}(t, \cdot)\right] \in \mathcal{H}$.

\section{Tempered numbers and point values}

Definition 4.1 The ring of tempered numbers is defined as

$$
\mathbf{h}=\mathbf{s}^{\prime} / \mathbf{s}
$$

The elements of $\mathbf{h}$ are called tempered numbers.

Let $\left(b_{n}\right) \in \mathbf{s}^{\prime}$, we will use $\left[b_{n}\right]$ to denote the equivalence class $\left(b_{n}\right)+\mathbf{s}$.

Lemma 4.1 a) Let $\iota: \mathbb{R} \rightarrow \mathbf{h}$ be the application

$$
\iota(a)=[a] .
$$

Then $\iota$ is an embedding.

b) $\mathcal{H}$ is a $\mathbf{h}$-module with the natural operations.

c) Let $\left[f_{n}\right] \in \mathcal{H}$ and $a \in \mathbb{R}$. Then $\left[f_{n}(a)\right] \in \mathbf{h}$. 
Proof: a) It is clear that $(a) \in \mathbf{s}^{\prime}$, then $\iota(a)=[a]$ is well defined. Assuming that $\iota(a)=0$, we have that $(a) \in \mathbf{s}$. In particular $\lim _{n \rightarrow \infty} n a=0$, it follows that $a=0$.

b) We have divided the proof into two parts. We first prove that for $\left(b_{n}\right) \in \mathbf{s}^{\prime}$ and $\left(f_{n}\right) \in \mathcal{H}_{\mathrm{s}^{\prime}}$ we have $\left(b_{n} f_{n}\right) \in \mathcal{H}_{\mathrm{s}^{\prime}}$. In fact, by definition there exists constants $E, F>0$ and $p, q \in \mathbb{N}_{0}$ such that

$$
\begin{aligned}
\left\|f_{n}\right\|_{m} & \leq E(n+1)^{p} \\
\left|b_{n}\right| & \leq F(n+1)^{q} .
\end{aligned}
$$

Combining these inequalities, we obtain

$$
\left\|b_{n} f_{n}\right\|_{m} \leq E F(n+1)^{p+q} .
$$

This proves that $\left(\left\|b_{n} f_{n}\right\|_{m}\right) \in \mathbf{s}^{\prime}$, thus $\left(b_{n} f_{n}\right) \in \mathcal{H}_{\mathbf{s}^{\prime}}$.

Finally, the proof is completed by showing that for $\left(a_{n}\right) \in \mathbf{s}$ and $\left(f_{n}\right) \in \mathcal{H}_{\mathbf{s}^{\prime}}$ or $\left(a_{n}\right) \in \mathbf{s}^{\prime}$ and $\left(f_{n}\right) \in \mathcal{H}_{\mathbf{s}}$ we have $\left(a_{n} f_{n}\right) \in \mathcal{H}_{\mathbf{s}}$.

c) Since $\delta_{a} \in \mathcal{S}^{\prime}$, there exists a constant $C>0$ and $m \in \mathbb{N}_{0}$ such that

$$
\left|f_{n}(a)\right|=\left|\delta_{a}\left(f_{n}\right)\right| \leq C\left\|f_{n}\right\|_{m},
$$

for all $n \in \mathbb{N}_{0}$.

Combining the above inequality with $\left(\left\|f_{n}\right\|_{m}\right) \in \mathbf{s}^{\prime}$ we conclude that $\left(f_{n}(a)\right) \in$ $\mathbf{s}^{\prime}$.

Remark 4.1 We observe that $\mathbf{h}$ is not a field, since there exist zero divisors in $\mathbf{h}$. In fact, $\left[1+(-1)^{n}\right],\left[1+(-1)^{n+1}\right] \in \mathbf{h}$ are non zero and its product is zero.

Definition 4.2 The point value of $\left[f_{n}\right] \in \mathcal{H}$ in $a \in \mathbb{R}$, denoted by $\left[f_{n}\right](a)$, is defined to be $\left[f_{n}(a)\right]$. 
Example 4.1 The point value of $\delta$ in $a \in \mathbb{R}$. From (12) we have that $\iota(\delta)(a)=\left[a_{n}\right]$, where

$$
a_{n}= \begin{cases}\frac{\sqrt{n+1}}{a} h_{n+1}(a) h_{n}(0) & \text { for } n \text { even }, \\ -\frac{\sqrt{n+1}}{a} h_{n}(a) h_{n+1}(0) & \text { for } n \text { odd } .\end{cases}
$$

Example 4.2 The point value of $x_{+}$in 0 . It is easy to check that $\iota\left(x_{+}\right)(0)=$ $\left[a_{n}\right]$, where

$$
a_{n}= \begin{cases}\sqrt{n+1} h_{n}(0) \int_{0}^{\infty} h_{n+1}(x) d x & \text { for } n \text { even }, \\ \sqrt{n} h_{n-1}(0) \int_{0}^{\infty} h_{n}(x) d x & \text { for } n \text { odd } .\end{cases}
$$

We introduce the concept of association for tempered numbers.

Definition 4.3 Let $\left[a_{n}\right]$ and $\left[b_{n}\right]$ be tempered numbers. We say that $\left[a_{n}\right]$ and $\left[b_{n}\right]$ are associated, denoted by $\left[a_{n}\right] \approx\left[b_{n}\right]$, if

$$
\lim _{n \rightarrow \infty}\left(a_{n}-b_{n}\right)=0 .
$$

We observe that the relation $\approx$ is well defined and that $\approx$ is an equivalence relation on $\mathbf{h}$.

Example $4.3 \iota\left(x_{+}\right)(0) \approx 0$.

\section{$5 \quad$ Integration and Fourier transform}

In this section we present the integration theory of tempered generalized functions and the Fourier transform.

Definition 5.1 Let $\left[f_{n}\right] \in \mathcal{H}$ and $A$ be a Lebesgue measurable set. The integral of $\left[f_{n}\right]$ on $A$, denoted by $\int_{A}\left[f_{n}\right](x) d x$ is defined to be

$$
\left[\int_{A} f_{n}(x) d x\right] .
$$


We observe that the integral is well defined as an element of $\mathbf{h}$. In fact, as $1_{A}$ is a tempered distribution there exists a constant $C>0$ and $m \in \mathbb{N}_{0}$ such that

$$
\left|\int_{A} g(x) d x\right| \leq C\|g\|_{m}
$$

for all $g \in \mathcal{S}$. In particular, for $\left[f_{n}\right] \in \mathcal{H}$ we have that

$$
\left|\int_{A} f_{n}(x) d x\right| \leq C\left\|f_{n}\right\|_{m}
$$

As $\left(\left\|f_{n}\right\|_{m}\right) \in \mathbf{s}^{\prime}$, we conclude that $\int_{A}\left[f_{n}\right](x) d x \in \mathbf{h}$.

In the next Lemma we collect some fundamental properties of the integral of tempered generalized functions.

Lemma 5.1 Let $\left[f_{n}\right]$ and $\left[g_{n}\right]$ be tempered generalized functions, $a=\left[a_{n}\right] \in \mathbf{h}$ and $\alpha \in \mathbb{N}$. Then

a) Let $A$ and $B$ disjoint Lebesgue measurable sets. Then

$$
\int_{A \cup B}\left[f_{n}\right](x) d x=\int_{A}\left[f_{n}\right](x) d x+\int_{B}\left[f_{n}\right](x) d x .
$$

b)

$$
\int_{A}\left(\left[f_{n}\right]+a\left[g_{n}\right]\right)(x) d x=\int_{A}\left[f_{n}\right](x) d x+a \int_{A}\left[g_{n}\right](x) d x .
$$

c) Let $\varphi \in \mathcal{S}$. Then

$$
\iota\left(\int_{A} \varphi d x\right)=\int_{A} \iota(\varphi) d x .
$$

d) "Rule of integration by parts"

$$
\int_{\mathbb{R}}\left[f_{n}\right] D^{\alpha}\left[g_{n}\right](x) d x=(-1)^{\alpha} \int_{\mathbb{R}}\left(D^{\alpha}\left[f_{n}\right]\right)\left[g_{n}\right](x) d x .
$$

e) Let $\varphi \in \mathcal{S}$ and $T \in \mathcal{S}^{\prime}$. Then

$$
\int_{\mathbb{R}} \iota(T) \cdot \iota(\varphi)(x) d x=\iota(T(\varphi)) .
$$


Proof: The proof of $\mathbf{a}), \mathbf{b}$ ) and $\mathbf{c}$ ) are immediate.

d) We have that

$$
\begin{aligned}
\int_{\mathbb{R}}\left[f_{n}\right] D^{\alpha}\left[g_{n}\right](x) d x & =\left[\int_{\mathbb{R}} f_{n} D^{\alpha} g_{n}(x) d x\right] \\
& =\left[\int_{\mathbb{R}}(-1)^{\alpha} D^{\alpha} f_{n}(x) g_{n}(x) d x\right] \\
& =(-1)^{\alpha} \int_{\mathbb{R}}\left(D^{\alpha}\left[f_{n}\right]\right)\left[g_{n}\right](x) d x .
\end{aligned}
$$

e) By definitions

$$
\int_{\mathbb{R}} \iota(T) \cdot \iota(\varphi)(x) d x=\left[\int_{\mathbb{R}} T_{n}(x) \varphi(x) d x\right]
$$

Let us prove that $\left(T(\varphi)-\int T_{n}(x) \varphi(x) d x\right) \in \mathbf{s}$. Combining definitions, the $\mathrm{N}$-representation theorem and $\lim _{n \rightarrow \infty}<\varphi, h_{j}>=0$, we obtain that there exists $n_{0} \in \mathbb{N}$ such that if $n \geq n_{0}$,

$$
\begin{aligned}
\left|T(\varphi)-\int_{\mathbb{R}} T_{n}(x) \varphi(x) d x\right| & =\left|T-T_{n}(\varphi)\right| \\
& \leq \sum_{j=n+1}^{\infty}\left|T\left(h_{j}\right)\right|<\varphi, h_{j}>\mid \\
& \leq \sum_{j=n+1}^{\infty} C(j+1)^{p}\left|<\varphi, h_{j}>\right| \\
& \leq \sum_{j=n+1}^{\infty} C(j+1)^{2 p}\left|<\varphi, h_{j}>\right|^{2} .
\end{aligned}
$$

Since $\varphi \in \mathcal{S}$, it follows that

$$
\lim _{n \rightarrow \infty} \sum_{j=n+1}^{\infty}(j+1)^{q}\left|<\varphi, h_{j}>\right|^{2}=0
$$

for all $q \in \mathbb{N}_{0}$. Combining the above inequalities we see that

$$
\lim _{n \rightarrow \infty}(n+1)^{r}\left|T(\varphi)-\int_{\mathbb{R}} T_{n}(x) \varphi(x) d x\right|=0,
$$

for all $r \in \mathbb{N}_{0}$. This proves that $\left(T(\varphi)-\int T_{n}(x) \varphi(x) d x\right) \in \mathbf{s}$, which completes the proof. 
Example 5.1 Let $T \in \mathcal{S}^{\prime}$. Then

$$
\begin{aligned}
\int_{\mathbb{R}} \iota(T)(x) d x & =\left[\int_{\mathbb{R}} T_{n}(x) d x\right] \\
& =\left[1\left(T_{n}\right)\right] \\
& =\left[\sum_{j \text { even }}^{n} T\left(h_{j}\right) h_{j}(0)(-1)^{\frac{j}{2}}\right],
\end{aligned}
$$

where the last equality follows from formula (7).

Example $5.2 \delta^{2}$. From formula (13) we have that

$$
\begin{aligned}
\int_{\mathbb{R}} \delta^{2}(x) d x & =\left[\int_{\mathbb{R}} \delta_{n}^{2}(x) d x\right] \\
& =\left[\frac{(n+1)}{\sqrt{2 \pi}} \frac{1}{2} \frac{3}{4} \cdots \frac{n-1}{n}\right] .
\end{aligned}
$$

The Fourier transform and the convolution are very important tools of classical and modern analysis, our aim is introduce these operations in the context of tempered generalized functions. We recall that the Fourier transform $\mathcal{F}: \mathcal{S} \rightarrow \mathcal{S}$ is defined by

$$
\mathcal{F}(\varphi)(t)=\frac{1}{\sqrt{2 \pi}} \int_{\mathbb{R}} e^{-i t x} \varphi(x) d x
$$

and the convolution $*: \mathcal{S} \times \mathcal{S} \rightarrow \mathcal{S}$ is defined by

$$
\varphi * \psi(t)=\int_{\mathbb{R}} \varphi(t-x) \psi(x) d x
$$

For a fuller treatment of the issues discussed below, we refer the reader to [19].

Definition 5.2 The Fourier transform of a generalized tempered function $\left[f_{n}\right]$, denoted by $\mathcal{F}\left(\left[f_{n}\right]\right)$, is defined to be $\left[\mathcal{F}\left(f_{n}\right)\right]$.

We observe that the above definition is independent of the representatives, because for all $m \in \mathbb{N}_{0}$ and $\varphi \in \mathcal{S}$ we have that $\|\mathcal{F}(\varphi)\|_{m}=\|\varphi\|_{m}$.

Here are some elementary properties of the Fourier transform and convolution. 
Theorem 5.1 a) The Fourier transform $\mathcal{F}: \mathcal{H} \rightarrow \mathcal{H}$ is a linear isomorphism and its inverse is given by

$$
\mathcal{F}^{-1}\left(\left[f_{n}\right]\right)=\left[\mathcal{F}^{-1}\left(f_{n}\right)\right]
$$

b) Let $\left[f_{n}\right] \in \mathcal{H}$ and $\alpha \in \mathbb{N}_{0}$. Then

$$
\begin{aligned}
\mathcal{F}\left(D^{\alpha}\left[f_{n}\right]\right) & =(i x)^{\alpha} \mathcal{F}\left(\left[f_{n}\right]\right) \\
\mathcal{F}\left(x^{\alpha}\left[f_{n}\right]\right) & =i^{\alpha} D^{\alpha} \mathcal{F}\left(\left[f_{n}\right]\right) .
\end{aligned}
$$

c) Let $T$ be a tempered distribution. Then $\iota(\mathcal{F}(T))=\mathcal{F}(\iota(T))$.

Proof: a) Define $\mathcal{G}: \mathcal{H} \rightarrow \mathcal{H}$ by $\mathcal{G}\left(\left[f_{n}\right]\right)=\left[\mathcal{F}^{-1}\left(f_{n}\right)\right]$. We observe that $\mathcal{G}$ is well defined, because for any $m \in \mathbb{N}_{0}$ and $\varphi \in \mathcal{S}$ we have $\|\varphi\|_{m}=\left\|\mathcal{F}^{-1} \varphi\right\|_{m}$. It is clear that $\mathcal{F} \circ \mathcal{G}=I_{\mathcal{H}}$ and $\mathcal{G} \circ \mathcal{F}=I_{\mathcal{H}}$.

b) and c). The proofs follows from the definitions and properties of the Fourier transform in $\mathcal{S}$.

Definition 5.3 Let $\left[f_{n}\right]$ and $\left[g_{n}\right]$ be tempered generalized functions. The convolution of $\left[f_{n}\right]$ and $\left[g_{n}\right]$, denoted by $\left[f_{n}\right] *\left[g_{n}\right]$, is defined to be $\mathcal{F}^{-1}\left(\sqrt{2 \pi} \mathcal{F}\left(\left[f_{n}\right]\right)\right.$. $\left.\mathcal{F}\left(\left[g_{n}\right]\right)\right)$.

Theorem 5.2 a) Let $\left[f_{n}\right],\left[g_{n}\right] \in \mathcal{H}$. Then

$$
\left[f_{n}\right] *\left[g_{n}\right]=\left[f_{n} * g_{n}\right]
$$

b) Let $\left[f_{n}\right],\left[g_{n}\right],\left[h_{n}\right] \in \mathcal{H}$ and $\alpha \in \mathbb{N}_{0}$. Then

$$
\begin{aligned}
{\left[f_{n}\right] *\left[g_{n}\right] } & =\left[g_{n}\right] *\left[f_{n}\right] \\
D^{\alpha}\left(\left[f_{n}\right] *\left[g_{n}\right]\right) & =\left(D^{\alpha}\left[f_{n}\right]\right) *\left[g_{n}\right] \\
\left(\left[f_{n}\right] *\left[g_{n}\right]\right) *\left[h_{n}\right] & =\left[f_{n}\right] *\left(\left[g_{n}\right] *\left[h_{n}\right]\right)
\end{aligned}
$$


c) Let $\left[f_{n}\right],\left[g_{n}\right] \in \mathcal{H}$. Then

$$
\begin{aligned}
\mathcal{F}\left(\left[f_{n}\right] \cdot\left[g_{n}\right]\right) & =\frac{1}{\sqrt{2 \pi}} \mathcal{F}\left(\left[f_{n}\right]\right) * \mathcal{F}\left(\left[g_{n}\right]\right) \\
\mathcal{F}\left(\left[f_{n}\right] *\left[g_{n}\right]\right) & =\sqrt{2 \pi} \mathcal{F}\left(\left[f_{n}\right]\right) \cdot \mathcal{F}\left(\left[g_{n}\right]\right)
\end{aligned}
$$

d) Let $T \in \mathcal{S}^{\prime}$ and $\varphi \in \mathcal{S}$. Then

$$
\iota(T) * \iota(\varphi) \approx \iota(T * \varphi) .
$$

Proof: a) The proof is a consequence of the above theorem and definitions. b) and $\mathbf{c}$ ) The proofs follows from the definitions and properties of the convolution in $\mathcal{S}$.

d) We have that for all $\psi \in \mathcal{S}$,

$$
\begin{aligned}
\lim _{n \rightarrow \infty}<\left(T_{n} * \varphi\right), \psi> & =T * \varphi(\psi) \\
& =\lim _{n \rightarrow \infty}<(T * \varphi)_{n}, \psi>.
\end{aligned}
$$

This shows that $\iota(T) * \iota(\varphi) \approx \iota(T * \varphi)$.

Example 5.3 The Fourier transform of $\delta$. By formula (6) we have that

$$
\mathcal{F}(\iota(\delta))=\left[\sum_{k=0}^{n}(-i)^{k} h_{k}(0) h_{k}(x)\right] .
$$

\section{Generalized Stochastic Calculus}

Let $\left(\Omega, \mathcal{F},\left\{\mathcal{F}_{t}: t \in[0, T]\right\}, \mathbb{P}\right)$ be a filtered probability space, which satisfies the usual hypotheses. For a recent account of stochastic calculus and notations we refer the reader to the book of Ph. Protter [15].

Definition 6.1 Let $\left[f_{n}\right] \in \mathcal{H}, X$ be a continuous jointly measurable process and $V$ be a finite variation process. We define the integral of $\left[f_{n}\right](X)$ in relation to $V$ from 0 to $t$, denoted by $\int_{0}^{t}\left[f_{n}\right]\left(X_{s}\right) d V_{s}$, to be

$$
\left[\int_{0}^{t} f_{n}\left(X_{s}\right) d V_{s}\right]
$$


It is clear that for each $\omega \in \Omega$ and $t \in[0, T]$ we have that $\left[\int_{0}^{t} f_{n}\left(X_{s}\right) d V_{s}(\omega)\right] \in$ h, because

$$
\left|\int_{0}^{t} f_{n}\left(X_{s}\right) d V_{s}(\omega)\right| \leq \sup _{x}\left|f_{n}(x)\right||V|_{t}(\omega)
$$

where $|V|_{t}(\omega)$ is the total variation of $V$ in $[0, t]$.

Definition 6.2 a) Let $\left[f_{n}\right] \in \mathcal{H}$ and $X$ be a random variable. We define the expectation of $\left[f_{n}\right](X)$, denoted by $\mathbb{E}\left(\left[f_{n}\right](X)\right)$, to be $\left[\mathbb{E}\left(f_{n}(X)\right)\right]$.

b) Let $\left[f_{n}\right] \in \mathcal{H}, X$ be a continuous jointly measurable process and $V$ be a finite variation process such that $|V|_{t}$ is integrable. We define the expectation of $\int_{0}^{t}\left[f_{n}\right]\left(X_{s}\right) d V_{s}$, denoted by $\mathbb{E}\left(\int_{0}^{t}\left[f_{n}\right]\left(X_{s}\right) d V_{s}\right)$, to be $\left[\mathbb{E}\left(\int_{0}^{t} f_{n}\left(X_{s}\right) d V_{s}\right)\right]$.

It is easy to check that the above notion is well defined. We also observe that the natural definition of expectation doesn't work. In fact, let $Y_{n}: \Omega \rightarrow \mathbb{R}$ be random variables such that $\left(Y_{n}(\omega)\right) \in \mathbf{s}^{\prime}$ for all $\omega \in \Omega$. We have that $\left[\left(\mathbb{E}\left(Y_{n}\right)\right)\right]$ is dependent of the representatives $\left(Y_{n}\right)$, because if $\left\{A_{n}: n \in \mathbb{N}\right\}$ is a partition measurable of $\Omega$ such that $\mathbb{P}\left(A_{n}\right)=\frac{1}{2^{n}}$ for all $n \in \mathbb{N}$ and $\left(b_{n}\right) \in \mathbf{s}^{\prime}$, $\left[Y_{n}(\omega)\right]=\left[Y_{n}(\omega)+b_{n} 2^{n} 1_{A_{n}}(\omega)\right]$ and $\left(\mathbb{E}\left(b_{n} 2^{n} 1_{A_{n}}\right)\right)=\left(b_{n}\right) \notin \mathbf{s}$.

We can now prove the Itô formula for tempered generalized functions. Clearly, this formula is an extension of the classical Itô formula via infinite dimensional methods.

Theorem 6.1 Let $\left[f_{n}\right] \in \mathcal{H}$ and $X$ be a continuous semimartingale. Then

$$
\left[f_{n}\right]\left(X_{t}\right)=\left[f_{n}\right]\left(X_{0}\right)+\int_{0}^{t} D\left[f_{n}\right]\left(X_{s}\right) d X_{s}+\frac{1}{2} \int_{0}^{t} D^{2}\left[f_{n}\right]\left(X_{s}\right) d<X>_{s}
$$

where $\int_{0}^{t} D\left[f_{n}\right]\left(X_{s}\right) d X_{s}(\omega)$ defined by $\left[\int_{0}^{t} D f_{n}\left(X_{s}\right) d X_{s}(\omega)\right]$ is the Itô integral of $\left[f_{n}\right](X)$ in relation to $X$ from 0 to $t$.

Proof: We first show that $\left[\int_{0}^{t} D f_{n}\left(X_{s}\right) d X_{s}(\omega)\right]$ is well defined. In fact, let $\left(g_{n}\right) \in \mathcal{H}_{\mathbf{s}}$. Since $\left(D g_{n}\right) \in \mathcal{H}_{\mathbf{s}}$, we have $\left(\int_{0}^{t} D g_{n}\left(X_{s}\right) d<X>_{s}(\omega)\right) \in \mathbf{s}$. 
We see that $\left(g_{n}\left(X_{t}(\omega)\right)\right)$ and $\left(g_{n}\left(X_{0}(\omega)\right)\right)$ are in $\mathbf{s}$, which is clear from the definition of point value. Combining these facts with the Itô formula we have

$\left(\int_{0}^{t} D g_{n}\left(X_{s}\right) d X_{s}(\omega)\right)=\left(g_{n}\left(X_{t}(\omega)\right)\right)-\left(g_{n}\left(X_{0}(\omega)\right)\right)-\left(\frac{1}{2} \int_{0}^{t} D^{2} g_{n}\left(X_{s}\right) d<X>_{s}(\omega)\right)$

are in $\mathbf{s}$. Finally we see that $\left[\int_{0}^{t} D f_{n}\left(X_{s}\right) d X_{s}(\omega)\right] \in \mathbf{h}$ and the formula (19) holds, this is clear from the Itô formula applied to $f_{n}$,

$f_{n}\left(\left(X_{t}(\omega)\right)\right)=f_{n}\left(\left(X_{0}(\omega)\right)\right)+\int_{0}^{t} D f_{n}\left(X_{s}\right) d X_{s}(\omega)+\frac{1}{2} \int_{0}^{t} D^{2} f_{n}\left(X_{s}\right) d<X>_{s}(\omega)$.

Remark 6.1 Let $f \in \mathcal{S}$. By Proposition [3.2, $[f]=\left[f_{n}\right]$. Then it is clear that

$$
\left[f_{n}\right]\left(X_{t}\right)=\left[f\left(X_{t}\right)\right]
$$

and

$$
\int_{0}^{t} D^{2}\left[f_{n}\right]\left(X_{s}\right) d<X>_{s}=\left[\int_{0}^{t} D^{2} f\left(X_{s}\right) d<X>_{s}\right] .
$$

Consequently,

$$
\int_{0}^{t} D\left[f_{n}\right]\left(X_{s}\right) d X_{s}=\left[\int_{0}^{t} D f\left(X_{s}\right) d X_{s}\right]
$$

In particular, the members of the Itô formula for $f$ as function are the same that the members of the Itô formula for $f$ as tempered generalized function.

Remark 6.2 We observe that the members of the Itô formula for $C^{4}$ functions with appropriated decreasing at infinite are associated with the corresponding members of the Itô formula as tempered generalized functions. In fact, we have that $\left(f_{n}\right)$ converges uniformly over compacts whenever $f$ is twice continuously differentiable and $O\left(e^{-c x^{2}}\right)$ for some $c>1$ as $x \rightarrow \infty$ (see [25] for more details). In particular,

$$
\left[f_{n}\right](x) \approx[f(x)]
$$


for all $x \in \mathbb{R}$. Thus

$$
\left[f_{n}\right]\left(X_{t}\right) \approx\left[f\left(X_{t}\right)\right] \text { and }\left[f_{n}\right]\left(X_{0}\right) \approx\left[f\left(X_{0}\right)\right]
$$

If $D^{2} f \in \mathcal{C}^{2}$ and $D^{2} f$ is $O\left(e^{-c x^{2}}\right)$ for some $c>1$ as $x \rightarrow \infty$ we have that

$$
\int_{0}^{t} D^{2}\left[f_{n}\right]\left(X_{s}\right) d<X>_{s} \approx\left[\int_{0}^{t} D^{2} f\left(X_{s}\right) d<X>_{s}\right] .
$$

Combining the above identities with the classical Itô formula for $f$ we conclude that

$$
\int_{0}^{t} D\left[f_{n}\right]\left(X_{s}\right) d X_{s} \approx\left[\int_{0}^{t} D f\left(X_{s}\right) d X_{s}\right]
$$

Remark 6.3 We recall that S. Ustunel [26] and B. Rajeev [17] obtained Itô formula for tempered distributions, in the context of stochastic integration in Hilbert spaces. Our formula (19) is different from the Itô formulas given in [17] and [26], in particular, the formulas in these references do not extend the classical Itô formula as ours does.

Corollary 6.2 Let $X$ be a semimartingale. Then

$$
\left|X_{t}-a\right|=\left|X_{0}-a\right|+\int_{0}^{t} \operatorname{sgn}\left(X_{s}-a\right) d X_{s}+\int_{0}^{t} \delta_{a}\left(X_{s}\right) d<X>_{s} .
$$

Proof: Applying the Itô formula (19) to the tempered distribution $|\cdot-a|$ we obtain (201).

Corollary 6.3 Let $\left[f_{n}\right] \in \mathcal{H}$ and $B$ be a Brownian motion such that $B_{0}=0$. Then

$$
\mathbb{E}\left(\left[f_{n}\right]\left(B_{t}+x\right)\right)=\left[f_{n}\right](x)+\frac{1}{2} \int_{0}^{t} \mathbb{E}\left(D^{2}\left[f_{n}\right]\left(B_{s}+x\right)\right) d s
$$

Proof: We have

$$
\mathbb{E}\left(\left[\int_{0}^{t} D\left[f_{n}\right]\left(B_{s}+x\right) d B_{s}\right]\right)=\left[\mathbb{E} \int_{0}^{t} D f_{n}\left(B_{s}+x\right) d B_{s}\right]=0,
$$

because $\int D f_{n}\left(B_{s}+x\right) d B_{s}$ is a martingale. 
Corollary 6.4 Let $\left[f_{n}\right] \in \mathcal{H}$. Then $g_{t}=\left[\mathbb{E}\left(f_{n}\left(B_{t}+\cdot\right)\right)\right] \in \mathcal{H}_{T}$ solves the Cauchy problem

$$
\begin{aligned}
D_{t} g & =\frac{1}{2} D_{x}^{2} g \\
g_{0} & =\left[f_{n}\right]
\end{aligned}
$$

Proof: We observe that

$$
\mathbb{E}\left(f_{n}\left(B_{t}+x\right)\right)=\int_{\mathbb{R}} f_{n}(y) p_{t}(x-y) d y=f_{n} * p_{t}(x)
$$

where $p_{t}(y)=\frac{1}{\sqrt{2 \pi t}} e^{-\frac{y^{2}}{2 t}}$ is the heat kernel. As $*$ is a continuous operation in $\mathcal{S}$ and $\lim _{n \rightarrow \infty} f_{n} * p_{t}=f_{n}$ in $\mathcal{S}$ we conclude that $\left[g_{n}\right] \in \mathcal{H}_{T}$ where the functions $g_{n}:[0, T] \times \mathbb{R} \rightarrow \mathbb{R}$ are given by $g_{n}(t, x)=\mathbb{E}\left(f_{n}\left(B_{t}+x\right)\right)$. Applying the formula (21) we completes the proof.

Theorem 6.5 Let $\left[f_{n}\right] \in \mathcal{H}_{T}$ and $X$ be a continuous semimartingale. Then

$$
\begin{aligned}
{\left[f_{n}\right]\left(X_{t}\right)=} & {\left[f_{n}\right]\left(X_{0}\right)+\int_{0}^{t} D_{t}\left[f_{n}\right]\left(s, X_{s}\right) d s+\int_{0}^{t} D_{x}\left[f_{n}\right]\left(s, X_{s}\right) d X_{s} } \\
& \left.+\frac{1}{2} \int_{0}^{t} D_{x}^{2}\left[f_{n}\right]\left(s, X_{s}\right) d<X\right\rangle_{s} .
\end{aligned}
$$

Proof: We observe that $\int_{0}^{t} D_{t}\left[f_{n}\right]\left(s, X_{s}\right) d s$ is well defined and proceed analogously to the proof of the extension of Itô formula.

\section{References}

[1] S. Albeverio, Z. Haba, F. Russo, A two-space dimensional semilinear heat equation perturbed by (Gaussian) white noise, Probab. Theory Related Fields 121(2001) 319-366.

[2] P. Antosik, J. Mikusiński, R. Sikorski, Theory of Distributions. The Sequential Approach, Elsevier Scientific Publishing Company, 1973. 
[3] H. Biagioni, A Nonlinear Theory of Generalized functions, Lectures Notes in Mathematics, Springer-Verlag, Berlin, 1421 (1990).

[4] U. Çapar, H. Aktuğlu, An overall view of stochastics in Colombeau related algebras, in : L. Decreusefond, B. Oksendal, S. Ustnel(Edis.), Stochastic analysis and related topics VIII, Progr. Probab, 2003, 6790 .

[5] J. Colombeau, Elementary Introduction to New generalized Functions, Noth-Holland Math Studies 113, Noth Holland, Amsterdam, 1985.

[6] P. Catuogno, S. Molina, C. Olivera, On Hermite representation of distributions and products, Integral Transforms and Special Functions. 18 (2007) 233-243.

[7] A. Delcroix Regular rapidly decreasing nonlinear generalized functions. Application to microlocal regularity., J. Math. Anal. Appl. 327 (2007) 564-584.

[8] H. Föllmer, Calcul d' Itô sans probabilités., Lecture Notes in Math. 850 (1981) 143-150.

[9] M. Grosser, M. Kunzinger, M. Oberguggenberger, R. Steinbauer, Geometric theory of generalized function with applications to general relativity., Kluwer Academic Publishers, Dordrecht, 2001.

[10] C. Martias, Extension du calcul d'Itô aux distributions de Schwartz, C. R. Acad. Sci. Paris Sr. I Math. 321 (1995) 331-334.

[11] C. Martias, Stochastic integration on generalized function spaces and its applications, Stochastics Stochastics Rep. 57 (1996) 289-301. 
[12] R. Mirkova, S. Pilipovich, D. Seleši, Generalized stochastic processes in algebras of generalized functions, J. Math. Anal. Appl. 353 (2008) 260-270.

[13] M. Oberguggenberger, Multiplication of Distributions and Applications to Partial Differential Equations, Pitman Research Notes in Math. Series 259. Longman, Harlow, 1993.

[14] M. Oberguggenberger, F. Russo, Nonlinear SPDEs: Colombeau solutions and pathwise limits, in : L. Decreusefond (Edi), Stochastic analysis and related topics, VI, Progr. Probab., 1998 319-332.

[15] Ph. Protter, Stochastic integration and differential equations., Second edition. Stochastic Modelling and Applied Probability, 21. SpringerVerlag, Berlin, 2005.

[16] Y. Radyno, N. Tkhan, S. Ramadan, The Fourier transformation in an algebra of new generalized functions, Acad. Sci. Dokl. Math. 46 (1993) 414-417.

[17] B. Rajeev, From Tanaka's formula to Ito's formula: distributions, tensor products and local times, Lectures Notes in Mathematics, SpringerVerlag, Berlin, 1775 (2001) 371-389.

[18] M. Reed, B. Simon, Methods of Modern Mathematical Physics. vol. 1, Academic Press, 1980.

[19] M. Reed, B. Simon, Methods of Modern Mathematical Physics. vol. 2, Academic Press, 1975.

[20] F. Russo, Colombeau generalized functions and stochastic analysis, Edit. A.l. Cardoso, M. de Faria, J Potthoff, R. Seneor, L. Streit, Stochastic analysis and applications in physics, NATO Adv. Sci. Inst. 
Ser. C Math. Phys. Sci., Kluwer Acad. Publ., Dordrecht, 449(1994) 329-249.

[21] S. Sadlok, On Hermite expansion of $x_{+}^{p}$, Annales Polonici Mathematici. XXXVIII (1980) 159-162.

[22] L. Schwartz, Théorie des distributions, Hermann, Paris, 1966.

[23] B. Simon, Distributions and Their Hermite Expansions, Journal of Math. Phys. 121 (1971) 140-148.

[24] C. Shen, M. Sun, On the neutral Hermite product of the distributions $x^{n}$ and $\delta^{(m)}(x)$, Integral Transforms Spec. Funct. 19 (2008) 387-398.

[25] M. Stone, Developments in Hermite polynomials, Ann. of Math. 29 (1927/1928) 1-13.

[26] S. Üstunel, A generalization of Itô formula, Journal of Funct. Anal. 47 (1982) 143-152. 